\title{
Expression of CD34 and $\beta$-Catenin in Malignant Rhabdoid Tumor of the Liver Mimicking Proximal-Type Epithelioid Sarcoma
}

\author{
Woo Cheal Cho $\cdot$ Fabiola Balarezo \\ Department of Pathology and Laboratory Medicine, Hartford Hospital, Hartford, CT, USA
}

\begin{abstract}
Malignant rhabdoid tumor (MRT) is a rare, aggressive malignant neoplasm often arising in the kidney in infants or young children. MRT was first described in 1978 as a rhabdomyosarcomatoid variant of Wilms tumor with unfavorable prognosis due to its morphologic resemblance to rhabdomyoblasts. ${ }^{1}$ Since the first description outside the kidney, MRTs have been described in almost every conceivable location in the body, including the central nervous system where it is commonly referred to as atypical teratoid/rhabdoid tumor. ${ }^{2}$ Involvement of the liver, however, is still exceedingly rare, with only fewer than 60 cases of primary MRT arising in the liver reported in the literature. Herein, we describe a rare case of MRT of the liver in a 1-year-old male infant with CD34 and $\beta$-catenin expression mimicking proximal-type epithelioid sarcoma (ES).
\end{abstract}

\section{CASE REPORT}

A 1-year-old male infant presented with a right-sided, nontender abdominal mass. Imaging studies revealed a heterogeneously enhancing mass within the liver, measuring $10.0 \times 10.0$ $\times 7.9 \mathrm{~cm}$, suspicious of hepatoblastoma. Liver biopsy showed a proliferation of malignant epithelioid cells with clear to amphophilic cytoplasm and small nucleoli (Fig. 1A-C). The tumor focally exhibited an organoid/trabecular growth pattern, and a rim of compressed non-neoplastic liver parenchyma was seen at

Corresponding Author

Woo Cheal Cho, MD

Department of Pathology and Laboratory Medicine, Hartford Hospital, 80 Seymour

Street, Hartford, CT 06102-5037, USA

Tel: +1-860-972-2488, Fax: +1-860-545-2204

E-mail: woocheal.cho@hhchealth.org

Received: March 12, 2017 Revised: April 5, 2017

Accepted: May 15, 2017 the periphery of the tumor (Fig. 1A). Necrosis was present (Fig. 1B) and numerous mitotic figures (7 mitoses/10 high-power field) (Fig. 1C) were identified. No definitive rhabdoid cells were seen. Immunohistochemical analysis revealed diffuse immunoreactivity to cytokeratin (CK) 19, CK MNF116, vimentin, and $\beta$-catenin (membranous) (Fig. 2A). The tumor was also focally positive for epithelial membrane antigen, glypican-3, and CD34 (Fig. 2B). Immunostains for CK7, CK20, hepatocyte paraffin (Hep Par) 1, arginase-1, $\alpha$-fetoprotein, desmin, myogenin, human melanoma black 45, Melan-A, octamer-binding transcription factor $3 / 4$, carcinoembryonic antigen, chromogranin, synaptophysin, calponin, smooth muscle actin, S100, and SRY-related HMG-box 10 (SOX10) were negative. Loss of nuclear INI1 expression (Fig. 2C) was seen. The diagnosis of INI1-negative neoplasm was entertained with differential diagnoses including extrarenal MRT, proximal-type ES, and small cell undifferentiated (SCUD) hepatoblastoma. Despite the lack of classic rhabdoid morphology and the presence of positive expression of CD34 and $\beta$-catenin, extrarenal MRT was favored over proximal-type ES given the patient's age and location of the tumor. In addition, the tumor lacked classic morphologic features of small round blue cell tumors, thereby making SCUD hepatoblastoma a less favored differential.

Formal written informed consent was not required with a waiver by the appropriate institutional review board of Hartford Hospital and/or national research ethics committee.

\section{DISCUSSION}

MRT is a rare, highly aggressive and lethal malignant neoplasm with poor prognosis. Histologically, MRT is classically characterized by sheets of large eosinophilic cells with eccentric 

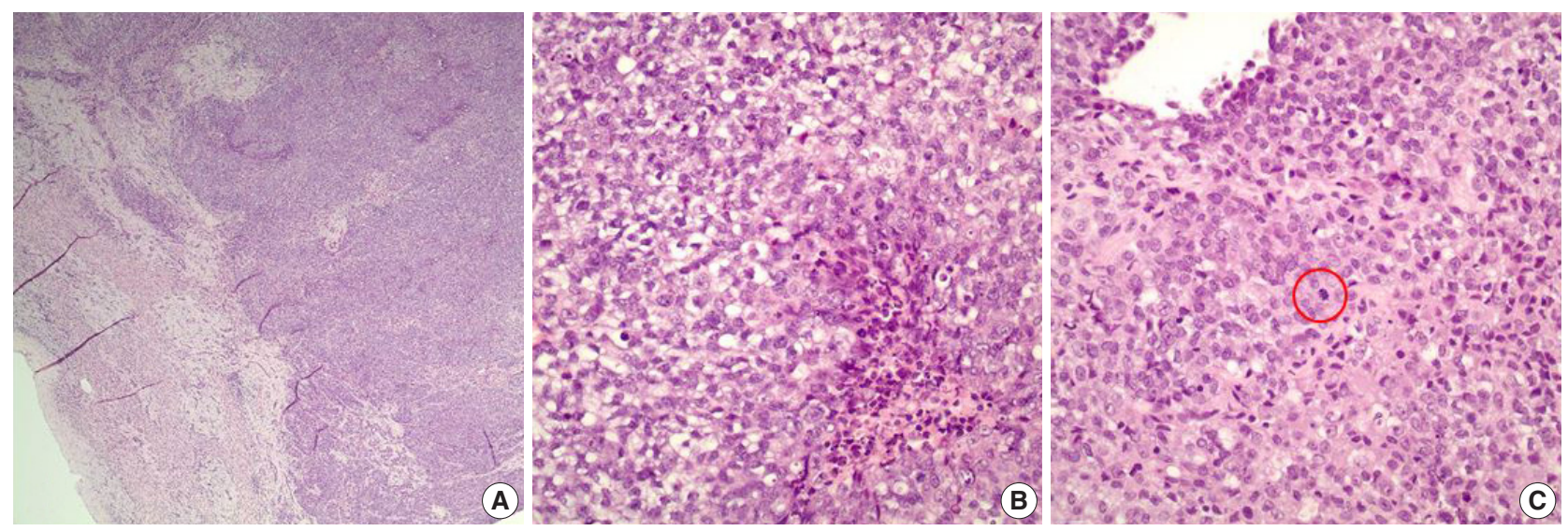

Fig. 1. Microscopic findings on liver biopsy. (A) The tumor displays malignant epithelioid cells with clear to amphophilic cytoplasm and small nucleoli with a focal organoid growth pattern. A rim of compressed non-neoplastic liver parenchyma is seen at the periphery of the tumor. (B, C) Areas of necrosis $(B)$ and frequent mitoses (C, circle) within the tumor are also seen.
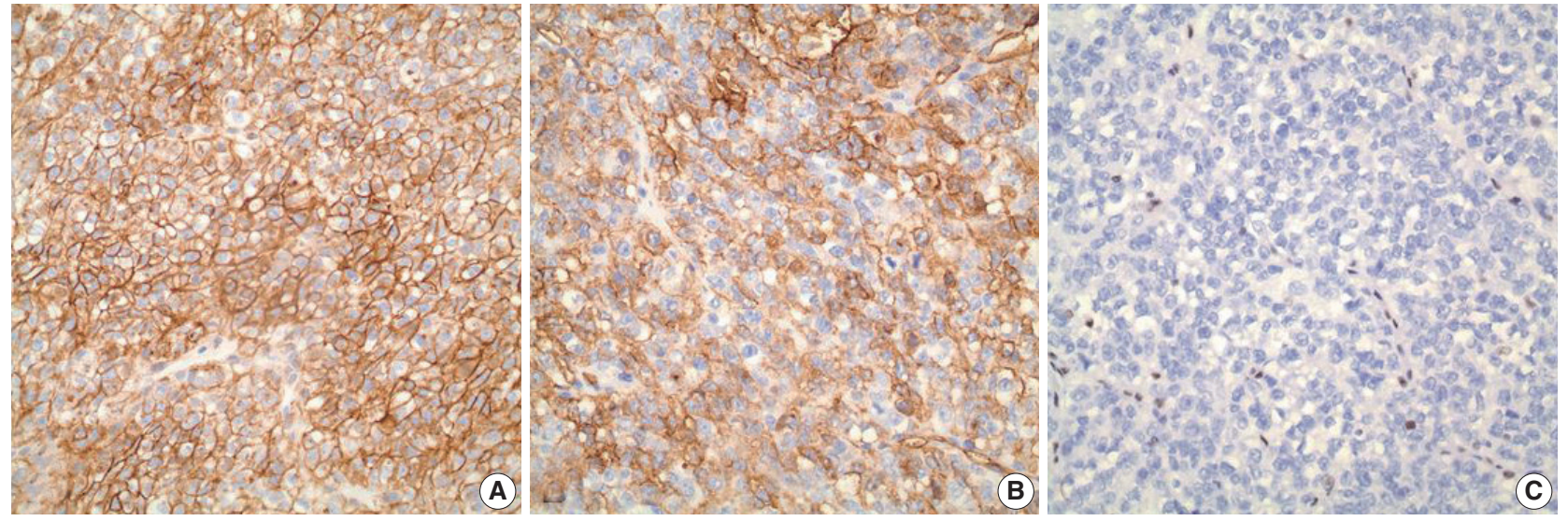

Fig. 2. Immunohistochemical analysis. (A) A diffuse and strong immunoreactivity (membranous) with $\beta$-catenin is seen in the tumor. (B) The tumor is also positive (patchy) for CD34. (C) Loss of nuclear INI1 expression is seen within the tumor.

vesicular nuclei, prominent nucleoli, and occasional intracytoplasmic inclusions of hyaline globules, reminiscent of rhabdomyoblasts. Loss of nuclear INI1 expression associated with deletions or mutations of the SMARCB1/INI1 gene at 22q11.2 is the characteristic hallmark of MRT., ${ }^{3,4}$ This genetic alteration, however, is also seen in other rare neoplasms, including proximaltype $\mathrm{ES}^{5}$ and SCUD hepatoblastoma. ${ }^{6}$ In particular, distinction between extrarenal MRT and proximal-type ES can be problematic due to morphologic similarities between the two entities; proximal-type ES also shows large epithelioid cells, vesicular nuclei with prominent nucleoli, and rhabdoid cytoplasmic inclusions. Recently, CD34 and $\beta$-catenin have been suggested as potentially useful immunohistochemical markers for distinguishing extrarenal MRT from proximal-type ES. Proximal-type ES often exhibits positive expression of $\mathrm{CD} 34$ and $\beta$-catenin, while extrarenal MRT typically lacks immunoreactivity to these markers. In the present case, however, the tumor was diffusely and strongly positive for $\beta$-catenin while showing patchy positivity with CD34 in the absence of nuclear INI1 staining, mimicking proximal-type ES. Nonetheless, extrarenal MRT was still favored over proximal-type ES in our case given the fact that proximal-type ES is predominantly seen in middle-aged or older adults, frequently occurring in axial or proximal regions, such as the pelvis, perineum, and genitalia. ${ }^{7}$ In contrast, MRT of the liver mainly occurs in infants. ${ }^{4}$ SCUD hepatoblastoma is a rare variant of hepatoblastoma known to show loss of INI expression, similar to MRT. ${ }^{6}$ In fact, some authors ${ }^{6}$ have recently postulated that SCUD hepatoblastoma may actually not be a hepatoblastoma but rather a form of MRT arising in the liver, although further study is needed. The tumor in our case, however, lacked small cell morphology compatible with SCUD hepatoblastoma, thus making it a less favored differential. 
In conclusion, we report a case of MRT of the liver with unusual immunophenotypic features mimicking proximal-type ES and SCUD hepatoblastoma. To the best of our knowledge, this may be the first reported case of extrarenal MRT of the liver with $\mathrm{CD} 34$ and $\beta$-catenin expression.

\section{ORCID}

Woo Cheal Cho: http://orcid.org/0000-0001-5867-1403

\section{Conflicts of Interest}

No potential conflict of interest relevant to this article was reported.

\section{REFERENCES}

1. Beckwith JB, Palmer NF. Histopathology and prognosis of Wilms tumors: results from the First National Wilms' Tumor Study. Cancer 1978; 41: 1937-48.
2. Rorke LB, Packer RJ, Biegel JA. Central nervous system atypical teratoid/rhabdoid tumors of infancy and childhood: definition of an entity. J Neurosurg 1996; 85: 56-65.

3. Oita S, Terui K, Komatsu S, et al. Malignant rhabdoid tumor of the liver: a case report and literature review. Pediatr Rep 2015; 7: 5578.

4. Oda Y, Tsuneyoshi M. Extrarenal rhabdoid tumors of soft tissue: clinicopathological and molecular genetic review and distinction from other soft-tissue sarcomas with rhabdoid features. Pathol Int 2006; 56: 287-95.

5. Hornick JL, Dal Cin P, Fletcher CD. Loss of INI1 expression is characteristic of both conventional and proximal-type epithelioid sarcoma. Am J Surg Pathol 2009; 33: 542-50.

6. Vokuhl C, Oyen F, Haberle B, von Schweinitz D, Schneppenheim $\mathrm{R}$, Leuschner I. Small cell undifferentiated (SCUD) hepatoblastomas: All malignant rhabdoid tumors? Genes Chromosomes Cancer 2016; 55: 925-31.

7. Armah HB, Parwani AV. Epithelioid sarcoma. Arch Pathol Lab Med 2009; 133: 814-9. 